\title{
Efficient 3D Model Retrieval using Cubic Spline Interpolation-Shape Distribution
}

\author{
Jingwei LI, Qiang CAI \& Haisheng LI \\ School of Computer and Information Engineering, Beijing Technology and Business University, Beijing, \\ China
}

\begin{abstract}
D model retrieval has been becoming a significant research field of modern information retrieval. To target at obtaining a better retrieval results, a novel Cubic Spline Interpolation-Shape Distribution algorithm (CSI-SD) is proposed. On the basis of traditional Shape Distribution algorithm (SD), The CSI-SD we propose improves the retrieval effect in two aspects: firstly, complete the normalization by gaining the 3D model's gravity center; secondly, use the cubic spline interpolation curve to characterize shape distribution histogram more accurately. We conduct experiments based on the large-scale comprehensive benchmark, which was compiled in SHREC2014. Experimental results show that our CSI-SD algorithm outperforms initial SD and the 3D Shape Histogram algorithm according to six general evaluation metrics.

KEYWORD: Shape Distribution; cubic spline interpolation; 3D model retrieval.
\end{abstract}

\section{INTRODUCTION}

With the rapid development of the threedimensional laser scanning technology and computer-assisted technology, there are a large number of 3D models spread online. Hence, 3D model retrieval is becoming a significant research field of modern information retrieval.

Currently, there are several kinds of retrieval algorithms, such as geometry-based algorithms, graph-based algorithms, view-based algorithms and so on. Geometry-based algorithms characterize the geometric information of a 3D model by describing the distribution of geometric elements and the feature extraction of them is usually designed with two goals: (1) Strong discriminative ability with respect to various 3D models; (2) The robustness to noise and distortion.

These geometry-based features can be divided into the global feature and the local feature. The global feature such as Shape Distribution (SD)[11] can be relatively effective at discriminating between two different 3D models, but there is still much room for improvement whatever in the respect of retrieval performance or retrieval time. Ankerst et al.[12] proposed a Shape Histogram algorithm, which is easier to understand and implement, but it has a relatively poor retrieval effect. The local feature can be listed as the 3D shape context[13], Conformal factor[6], and Poisson histogram descriptor[3]. The commonly used retrieval methods before 2008 can be referred in [16]. Recently, Zou et al.[7] proposed a combined shape distribution descriptor based on principal plane analysis and group integration, which also improves the total performance of shape distribution algorithm.

\section{CUBIC SPLINE INTERPOLATION-SHAPE DISTRIBUTION ALGORITHM}

The nature of the Osada et al.'s Shape Distribution algorithm (SD)[11] describe the 3d model's global geometric features by designing a shape function, then represent the object's signature as a shape distribution sampled. However it's way of normalized still could be improved and the way of describing shape distribution is not accurate enough.

Our CSI-SD has two main characteristics compared to SD: firstly, we normalize the $3 \mathrm{~d}$ model by shifting $3 \mathrm{~d}$ models' gravity center and scaling it directly; after that, to have a better describe with the shape distribution histogram, the cubic spline interpolation curve is used to represent the shape distribution.

\section{$2.13 \mathrm{D}$ models randomly sample points}

The initial SD is invariant to the translation and rotation actually, but it is sensitive to scale invariant. So SD normalizes the $3 \mathrm{~d}$ model by next three steps: (1) Align the maximum sample values; (2) align the 
mean sample values (or similarly the median); (3) search for the scale that produces the minimal dissimilarity measure during each comparison: firstly, we scale the distributions so that the mean sample in each distribution has value 1 . Then we can get shape distribution $f$ and $s$, similarity distances by using the formula $\min _{s} D(f(x), s g(s x))$, where $s$ is 100 values by calculate the formula $\log s=-10,-9.8, \cdots, 10$.

The first two normalized ways may not produce the minimal dissimilarity measures because of mismatching scales. The third way can gain the invariance in scale by using the minimum similarity distance of two shape distributions, however it has a large computational cost and low time efficiency. So we propose the following normalize way: firstly, by accumulating the gravities of all the faces on the surface, we can obtain a model's gravity center, then, we shift the gravity center to the origin, make the radius of its bounding sphere to be 1 .Therefore, the D2 distance feature value is compressed into the range of $[0,2]$, in the respect of scale invariance property it contributes to our algorithm. Finally, we randomly sample 1024 sample points for each model. The figure 1 shows examples.

\begin{tabular}{|c|c|c|c|}
\hline Models & Ant & Bee & Cup \\
\hline $\begin{array}{c}\text { Original } \\
\text { 3D }\end{array}$ & & \\
models' & \\
Vertices & & & \\
\hline $\begin{array}{c}\text { Normalized } \\
\text { 3D models' } \\
\text { Sample } \\
\text { point set }\end{array}$ & & & \\
\hline
\end{tabular}

Figure 1. Example sample point sets for normalized 3D models.

\subsection{Construct a cubic spline interpolation curve}

When describes the statistical shape distribution histogram, a usual method is polynomial fitting based on least square method. This method is simple and easy to achieve, but it is limited by using a polynomial to express the properties of curves, and the histogram's shape and tendency is ever-changing, so there is still room for improvement in the respect of describing the shape distribution histogram feature.

Considering the above problems, we adopt cubic spline interpolation curve [8] to describe the statistical shape histogram. Cubic spline interpolation curve is one of global fitting, which can describe the global feature of shape distribution precisely, and the feature owns both the interpolation property and fitting property, which is adapted to the characteristics of the random sampling points. The curve can be constructed as follows.

We denote the point set as $\left\{Q_{k}\right\}$, which converts from the shape distribution histogram. And $k=0,1,2 \cdots, n$, where $\mathrm{n}$ is the number of the histogram's bins, then

$$
C(u)=\sum_{i=0}^{n+2} N_{i, p}(u) P_{i}
$$

Where $C(u)$ represents $p$ th-degree spline curve; and $N_{i, p}(u)$ are the $p$ th-degree spline basis function in the $i$ th bin. When $p=3, C(u)$ is $c^{2}$ continuous cubic spline interpolation curve.

The contrast performance between polynomial fitting (the degree of polynomial is set as 3) and cubic spline interpolation curve is illustrated in figure 2. We can achieve that cubic spline interpolation curve could describe shape distribution more precisely.

\section{EXPERIMENTS}

All the algorithms discussed above are implemented by hybrid programmed with Matlab and $\mathrm{C}++$ language (Matlab 2012b and VS2010). All experiments are running in a computer with an Intel(R) CPU:

Xeon(R) E5620@2.40 GHz and 12.0GB of RAM. OS: Windows 7, 64-bit.

In the experiments we will use the extended large-scale 3D model retrieval benchmark (LSB) and six kinds of general evaluation metrics.

\subsection{Unified large scale benchmark: LSB}

The extended large-scale 3D model retrieval benchmark (LSB) is motivated by the large collection of human-drawn sketches built by Eitz et al.[2], it contains 171 classes and 8987 3D models. Every model is saved in a text file with the '.OFF' format.

The LSB is composed with eight $3 \mathrm{~d}$ model benchmarks, among them are the Princeton Shape Benchmark (PSB)[4], Toyohashi Shape Benchmark (TSB)[9], SHREC'12 Generic Track Benchmark (SHREC12GTB)[10], and Konstanz 3D Model Benchmark (CCCC)[14] belong to the Generic 3D model retrieval benchmarks; the Watertight Model Benchmark (WMB) [1], Bonn's Architecture Benchmark (BAB) [15], McGill 3D Shape Benchmark (MSB)[17], and Engineering Shape Benchmark (ESB)[5] belong to Specialized 3D model retrieval benchmarks. In the sense that the LSB is based on carefully compiled and freely available content. 

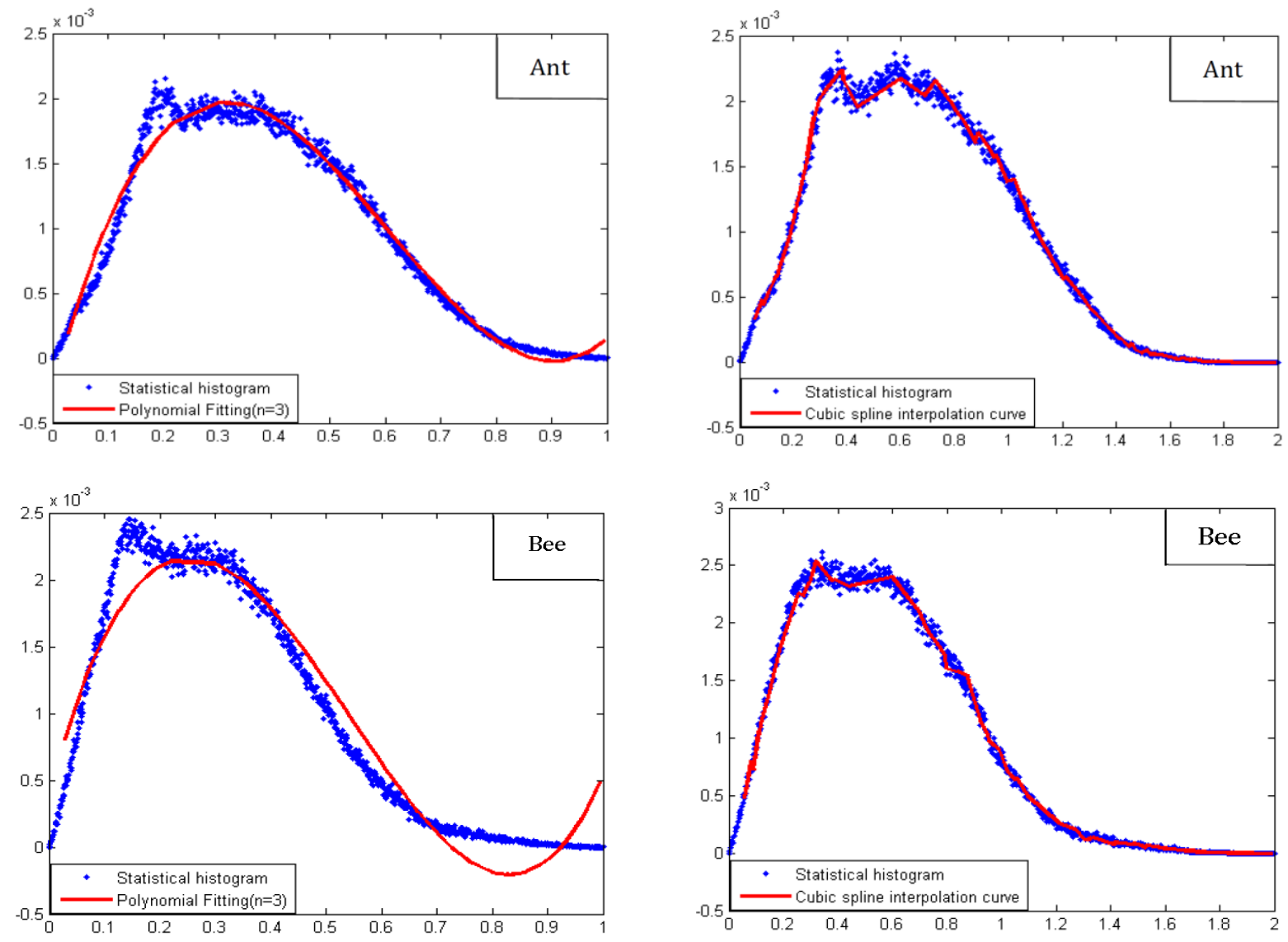

Figure 2. Contrast effects of shape distribution histogram between using polynomial fitting and cubic spline interpolation curve.

\subsection{General evaluation metrics}

To perform a comprehensive evaluation of a retrieval algorithm, we employed six performance metrics[4] which is commonly used in the 3D model retrieval field. They are First Tier (FT), Nearest Neighbor (NN), E-Measures (E), Second Tier (ST), Discounted Cumulated Gain (DCG) and PrecisionRecall (PR) diagram. The definitions are listed as follows.

- Precision-Recall plot (PR): We assume that there are $\mathrm{n}$ models, precision $\mathrm{P}$ is to measure the accuracy of relevant models among the top $\mathrm{K}(1 \leq K \leq n)$ ranking results, while recall $\mathrm{R}$ is the percentage of relevant class that has been retrieved in the top $\mathrm{K}$ ranking results.

- Nearest Neighbor (NN): $\mathrm{NN}$ is the precision of the top most model.

- First Tier (FT): Assume there are $\mathrm{C}$ relevant models, FT is the recall of the top C-1 retrieved models.

- Second Tier (ST): ST is the recall of the top 2(C-1) retrieved models.

- E-Measure (E): E-Measure is defined to measure the composite retrieval performance of both precision and recall of the top 32 retrieved models:

$$
E=\frac{2}{1 / P+1 / R}
$$

- Discounted Cumulated Gain (DCG): DCG is defined as the normalized summed weighted value. Firstly, the retrieval list $\mathrm{R}$ is transformed into a vector $\mathrm{G}$, if $R_{i}$ is a relevant model then $G_{i}=1$, otherwise $G_{i}=0$. DCG is computed as the following:

$$
D C G=\left\{\begin{array}{lr}
G_{1} & i=1, \\
D C G_{i-1}+\frac{G_{i}}{\log _{2} i} & \text { otherwise. }
\end{array}\right.
$$

And then, it is normalized by its optimum:

$$
D C G=\frac{D C G_{n}}{1+\sum_{j=2}^{C} \frac{1}{\log _{2} j}}
$$

\subsection{Result}

We choose the classes like cups, chairs, buses, beds and ants as query objects. When implementing SD and CSI-SD, here we all use D2 as shape function, and choose the parameters as follows: $N=1024$ samples, $B=1024$ bins and $V=64$, which means that we sample $N=1024$ sample points, divide the histogram into 1024 bins and make the sample points on shape distribution curve is $V=64$. As for the normalize way of SD, we choose the maximum of sampling distance in this experiment, in the meantime we use the polynomial curves fitting to construct shape distribution. The retrieval effects are shown in figure 3 and table 1. 


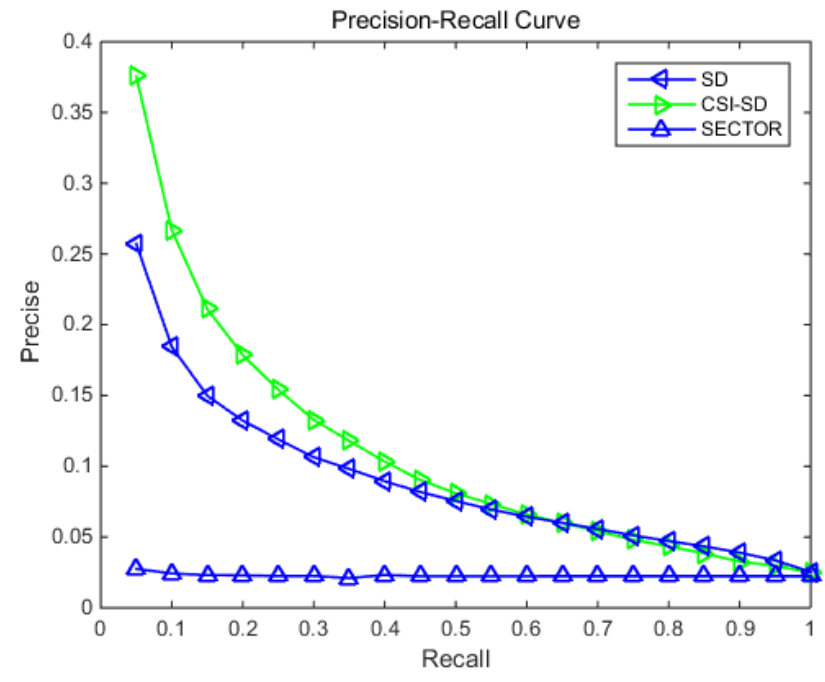

Figure 3. Precision-Recall plot performance comparisons on three different algorithms.

Table 1: five evaluation metrics between SD and CSI-SD

\begin{tabular}{|c|c|c|c|c|c|}
\hline Algorithm & NN & FT & ST & E & DCG \\
\hline SD & 0.22 & 0.11 & 0.17 & 0.07 & 0.55 \\
\hline SECTOR & 0.39 & 0.06 & 0.11 & 0.04 & 0.50 \\
\hline CSI-SD & 0.51 & 0.14 & 0.20 & 0.09 & 0.57 \\
\hline
\end{tabular}

Analysis the figure 3 and table 1 above we can know that our CSI-SD is superior to SD and the original classic algorithm SECTOR[2] with these six general evaluation metrics. But unfortunately that the mean time of SD is 4.00 seconds, SECTOR is 3.29 seconds and CSI-SD is 4.10 seconds, we have no advantage in terms of retrieval time.

\section{CONCLUSION}

The contribution of this paper is that we proposed a geometry-based technique which is named CSI-SD. It achieves two improvements on SD algorithm: (1) improved the method of normalized way; (2) use the cubic spline interpolation curve constructs shape distribution. Our algorithm owns a better retrieval effect from the experiments above. Of course, we also know that there is still plenty room, such as retrieval time, for improvement.

\section{REFERENCES}

[1] Tatsuma, H. Koyanagi, M. Aono. 2012. A large-scale shape benchmark for 3D object retrieval: Toyohashi Shape Benchmark, in: Proc. of 2012 Asia Pacific Signal and Information Processing Association (APSIPA2012).

[2] Li, A. Godil, M. Aono, X. Bai, T. Furuya, L. Li, R.J. López-Sastre, H. Johan, R.Ohbuchi, C. RedondoCabrera, A. Tatsuma, T. Yanagimachi, S. Zhang. 2012. SHREC'12 track: Generic 3D shape retrieval, in: $M$. Spagnuolo, M.M.B ronstein, A.M. Bronstein, A. Ferreira, (Eds.), 3DOR, Eurographics Association, pp. 119-126.

[3] Vranic. 2004. 3D Model Retrieval, PhD thesis, University of Leipzig.

[4] J. W. H. Tangelder, R. C. Veltkamp. 2008. A survey of content based 3D shape retrieval methods. Multimedia Tools and Applications, 39(3): 441-471.

[5] K.S. Huang, M.M. Trivedi. 2005. 3D shape context based gesture analysis integrated with tracking using omni video array, in: Proceedings of 2005 IEEE Computer Society Conference on Computer Vision and Pattern Recognition, p.80.

[6] K. Siddiqi, J. Zhang, D. Macrini, A. Shokoufandeh, S. Bouix, S. J. Dickinson. 2008. Retrieving articulated 3-D models using medial surfaces. Machine Vision Application, 19(4): 261-275.

[7] K. Zou, W.-H. Ip, C.-H. Wu, Z. Chen, K.-L. Yung, C.-Y. Chan. 2014. A novel 3D model retrieval approach using combined shape distribution, Multimedia Tools Appl. 69(2014)799-818.

[8] Les Piegl. 1997. The NURBS Book: Second Edition. Germany: Springer Verlag.

[9] M. Ankerst, G. Kastenmüller, H.-P. Kriegel, T. Seidl. 1999. 3D shape histograms for similarity search and classification in spatial databases. Lecture Notes in Computer Science, 1651: 207-22

[10] M. Ben-Chen, C. Gotsman. 2008. Characterizing shape using conformal factors, in: Eurographics Workshop on 3D Object Retrieval (3DOR), pp.1-8.

[11] M. Eitz, J. Hays, M. Alexa. 2012. How do humans sketch objects. ACM Trans. Graph, 31(4): 44:1-44:10.

[12] P. Shilane, P. Min, M. MKazhdan, T. A. Funkhouser. 2004. The Princeton shape benchmark, in: SMI, pp. 167178.

[13] R. C. Veltkamp, F. B. ter Haar. SHREC 2007 3D retrieval contest. 2007. Utrecht University: Department of Information and Computing Sciences, Technical Report: UU-CS-2007-015, 2007.

[14] R. Osada, T. Funkhouser, B. Chazelle, D. Dobkin. 2001. Matching 3D models with shape distributions// Proceedings of the Shape Modeling and Applications, Princeton, USA, 154-166.

[15] R. Wessel, I. Blümel, R. Klein. 2009. A 3D shape benchmark for retrieval and automatic classification of architectural data// Proceedings of the Eurographics Workshop on 3D Object Retrieval. Munich, Germany: 53-56.

[16] S. Jayanti, Y. Kalyanaraman, N. Iyer, K. Ramani. 2006. Developing an engineering shape benchmark for CAD models. Computer-Aided Design, 38(9): 939-953

[17] X. Pan, Q. You, Z. Liu, Q.H. Chen. 2011. 3D shape retrieval by poisson histogram, Pattern Recognition Letters, 32(6): 787-794. 